\title{
In-vitro activity of OPC-17116 against more than 6000 consecutive clinical isolates: a multicentre international study
}

\author{
F. Marco", R. N. Jones, D. J. Hoban', A. C. Pignatari', N. Yamane ${ }^{e}$ and R. Freif \\ ${ }^{a}$ Microbiology Laboratory, Hospital Clinic, University of Barcelona, Villarroel 170, \\ 08036-Barcelona, Spain; ${ }^{b}$ Department of Pathology, 5232 RCP, University of Iowa \\ Hospitals and Clinics, Iowa City, Iowa 52242, USA; ' Department of Clinical \\ Microbiology, University of Manitoba, Health Sciences Centre, MS675G-802 \\ Sherbrook Street, Winnipeg, Manitoba R3A 1R9, Canada; ${ }^{d}$ Laboratorios de Pesquisa \\ da Disciplina de Doenças Infecciosas, Escola Paulista de Medicina, Rua Botucatu 740 \\ CEP 04023, Sao Paulo, Brazil; 'Department of Laboratory Medicine, Kumamoto \\ University Medical School, 1-1-1, Honjo, Kumamoto 860, Japan, ${ }^{f}$ Kantonsspital Basel, \\ Bacteriology Laboratory, Petersgraben 4, CN-4032 Basel. Switzerland
}

\section{Introduction}

Fluoroquinolone antimicrobial agents have high activity against Gram-negative and Gram-positive bacteria, and are widely used for the treatment of a variety of bacterial infections. However, there is continued interest to develop new quinolones with improved antibacterial activity against Gram-positive organisms or with better pharmacokinetic profiles (Richard \& Gutmann, 1992). OPC-17116 is a new fluorinated quinolone with 1-cyclopropyl, 5-methyl and 7-methylpiperazino substituents (Yokota, Arai \& Kanda, 1991). Its activity against Enterobacteriaceae and Pseudomonas aeruginosa is comparable to current fluoroquinolones but, as has been observed with other newly synthesized quinolones, it seems to be more active against Gram-positive organisms (Imalda et al., 1992).

In this report, we studied the antibacterial activity of OPC-17116 against more than 6000 consecutive, non-fastidious, clinical isolates from six countries and compared its activity with those of ciprofloxacin and temafloxacin.

\section{Centres Participating}

\section{Materials and methods}

The six centres participating in the study were Hospital Clinic, University of Barcelona, Barcelona, Spain; University of Iowa Hospitals and Clinics, Iowa City, Iowa, USA; University of Manitoba, Health Sciences Centre, Winnipeg, Manitoba, Canada; Escola Paulista de Medicina, Sao Paulo, Brazil; Kumamoto University Medical School, Kumamoto, Japan and Kantonsspital Basle, Basel, Switzerland.

\section{Antimicrobial agents}

The drugs used in this study were OPC-17116 (Otsuka America Pharmaceuticals, Rockville, MD, USA); ciprofloxacin (Miles Inc., West Haven, CT, USA); temafloxacin 
(Abbott Pharmaceuticals, North Chicago, IL, USA); oxacillin (Bristol-Myers Squibb, Wallingford, CT, USA).

\section{Bacterial strains}

Each study centre tested at least 1000 consecutive, non-fastidious recent clinical isolates. Repeat isolates from the same patient were excluded to avoid testing of multiple copies of the same strain.

\section{MIC determinations}

MICs were determined by a broth microdilution method (NCCLS, 1990). Microdilution trays were prepared by each laboratory except the USA and Brazil participants who used a common tray lot produced in the USA. The test medium was cation-adjusted Mueller-Hinton broth. Oxacillin was used at a screening concentration $(2 \mathrm{mg} / \mathrm{L})$ in $2 \% \mathrm{NaCl}$ broth. The reference strains used in the study were as follows: Escherichia coli ATCC 25922, P. aeruginosa ATCC 27853, Staphylococcus aureus ATCC 29213 and Enterococcus faecalis ATCC 29212. The quality control organisms were tested weekly and results immediately sent by fax to the coordinating laboratory (University of Iowa Hospitals and Clinics) to assure that the NCCLS guidelines were followed and to confirm the validity of the data. Each participating centre was also requested to send the first 20 ciprofloxacin resistant Enterobacteriaceae strains (MIC $>2 \mathrm{mg} / \mathrm{L}$ ) detected during the study to the coordinating laboratory for additional investigation.

\section{Results and discussion}

The activities of OPC-17116, ciprofloxacin, and temafloxacin against Gram-negative and Gram-positive bacteria are given in Tables I and II, respectively. Against members of the Enterobacteriaceae family, activity of OPC-17116 was equal to or slightly lower than ciprofloxacin. At $\leqslant 0.03-0.5 \mathrm{mg} / \mathrm{L}$, OPC- 17116 inhibited $90 \%$ of the isolates $\left(\mathrm{MIC}_{90}\right)$ of most species, whereas for Enterobacter, Serratia and Providencia spp., OPC-17116 $\mathrm{MIC}_{90}$ s ranged from 0.25 to $>4 \mathrm{mg} / \mathrm{L}$. All the strains of other Enterobacteriaceae tested (11 species) were susceptible to OPC-17116 (MIC 90 $0.12 \mathrm{mg} / \mathrm{L}$ ). Resistance to ciprofloxacin in Enterobacteriaceae was detected in several species. Spain (E. coli, 20 strains) and Japan (Providencia rettgeri, eight strains; Providencia stuartii, three strains; and Serratia marcescens, seven strains) were the countries with the greatest numbers of resistant isolates, whereas the USA, Canada, Brazil and Switzerland isolated either no, or no more than five resistant strains. All but one ciprofloxacin-resistant strain were also resistant to OPC-17116 (MIC $>4 \mathrm{mg} / \mathrm{L})$. The remaining strain had an OPC- $17116 \mathrm{MIC}$ of $4 \mathrm{mg} / \mathrm{L}$ which was intrepreted to indicate the strain belonged to an intermediate category according to the NCCLS guidelines for other currently available fluoroquinolones (NCCLS, 1990). In this study, activity of OPC-17116 against members of the family Enterobacteriaceae was similar to activities reported in previous studies (Imada et al., 1992; Sader, Erwin \& Jones, 1992). However, we found a higher number of ciprofloxacin-resistant bacterial isolates that were also resistant to OPC-17116. Rates of resistance to fluoroquinolones seem to vary from country to country. In a recent national survey conducted in the USA (Jones et al., 1992) resistance to fluoroquinolones was relatively uncommon. In our study, the 
Table I. MICs (mg/L) of OPC-17116, ciprofloxacin and temafloxacin against Gram-negative clinical isolates

\begin{tabular}{|c|c|c|c|c|c|}
\hline $\begin{array}{l}\text { Organism } \\
\text { (no. of isolates) }\end{array}$ & $\begin{array}{l}\text { Antimicrobial } \\
\text { agent }\end{array}$ & $\mathrm{MIC}_{s 0}$ & $\mathrm{MIC}_{90}$ & Range & $\begin{array}{c}\text { Susceptibility } \\
(\%)\end{array}$ \\
\hline $\begin{array}{l}\text { Citrobacter diversus } \\
\text { (21) }\end{array}$ & $\begin{array}{l}\text { OPC-17116 } \\
\text { ciprofloxacin } \\
\text { temafloxacin }\end{array}$ & $\begin{array}{l}\leqslant 0 \cdot 03 \\
\leqslant 0.015 \\
\leqslant 0.03\end{array}$ & $\begin{array}{c}\leqslant 0.03 \\
\leqslant 0.015 \\
0.06\end{array}$ & $\begin{array}{l}\leqslant 0.03 \\
\leqslant 0.015 \\
\leqslant 0.03-0.06\end{array}$ & $\begin{array}{l}100 \\
100 \\
100\end{array}$ \\
\hline $\begin{array}{l}\text { Citrobacter freundii } \\
\text { (86) }\end{array}$ & $\begin{array}{l}\text { OPC-17116 } \\
\text { ciprofloxacin } \\
\text { temafloxacin }\end{array}$ & $\begin{array}{l}0.06 \\
0-03 \\
0.12\end{array}$ & $\begin{array}{l}0-5 \\
0 \cdot 25 \\
1\end{array}$ & $\begin{array}{l}\leqslant 0.03-4 \\
\leqslant 0.015->2 \\
\leqslant 0.03->4\end{array}$ & $\begin{array}{l}97 \\
99 \\
94\end{array}$ \\
\hline $\begin{array}{l}\text { Enterobacter } \\
\text { aerogenes }(80)\end{array}$ & $\begin{array}{l}\text { OPC- } 17116 \\
\text { ciprofloxacin } \\
\text { temafloxacin }\end{array}$ & 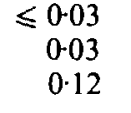 & $\begin{array}{l}0.25 \\
0.25 \\
0.5\end{array}$ & $\begin{array}{l}\leqslant 0.03-1 \\
\leqslant 0.015-1 \\
\leqslant 0.03-1\end{array}$ & $\begin{array}{l}100 \\
100 \\
100\end{array}$ \\
\hline $\begin{array}{l}\text { Enterobacter } \\
\quad \text { cloacae (244) }\end{array}$ & $\begin{array}{l}\text { OPC-17116 } \\
\text { ciprofloxacin } \\
\text { temafloxacin }\end{array}$ & 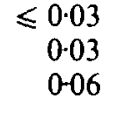 & $\begin{array}{l}0.5 \\
0.25 \\
0.5\end{array}$ & $\begin{array}{l}\leqslant 0.03->4 \\
\leqslant 0.015->2 \\
\leqslant 0.03->4\end{array}$ & $\begin{array}{l}98 \\
96 \\
97\end{array}$ \\
\hline $\begin{array}{l}\text { Enterobacter } \\
\quad \text { sakazakii }(11)\end{array}$ & $\begin{array}{l}\text { OPC-17116 } \\
\text { ciprofloxacin } \\
\text { temafloxacin }\end{array}$ & 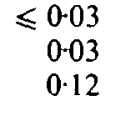 & $\begin{array}{r}4 \\
> \\
4\end{array}$ & $\begin{array}{l}\leqslant 0.03-4 \\
\leqslant 0015->2 \\
\leqslant 0.03->4\end{array}$ & $\begin{array}{l}82 \\
82 \\
82\end{array}$ \\
\hline $\begin{array}{l}\text { Enterobacter spp. } \\
\text { (33) }\end{array}$ & $\begin{array}{l}\text { OPC-17116 } \\
\text { ciprofloxacin } \\
\text { temafloxacin }\end{array}$ & $\begin{array}{l}0 \cdot 12 \\
0.06 \\
0 \cdot 12\end{array}$ & $\begin{array}{r}4 \\
>2 \\
>\end{array}$ & $\begin{array}{l}\leqslant 0.03->4 \\
\leqslant 0.015->2 \\
\leqslant 0.03->4\end{array}$ & $\begin{array}{l}82 \\
79 \\
79\end{array}$ \\
\hline E. coli $(1381)$ & $\begin{array}{l}\text { OPC-17116 } \\
\text { ciprofloxacin } \\
\text { temafloxacin }\end{array}$ & $\begin{array}{l}\leqslant 0 \cdot 03 \\
\leqslant 0 \cdot 015 \\
\leqslant 0.03\end{array}$ & $\begin{array}{l}0.12 \\
0.12 \\
0 \cdot 25\end{array}$ & $\begin{array}{l}\leqslant 0.03->4 \\
\leqslant 0.015->2 \\
\leqslant 0.03->4\end{array}$ & $\begin{array}{l}97 \\
97 \\
96\end{array}$ \\
\hline $\begin{array}{l}\text { Klebsiella oxytoca } \\
\quad(97)\end{array}$ & $\begin{array}{l}\text { OPC- } 17116 \\
\text { ciprofloxacin } \\
\text { temafloxacin }\end{array}$ & $\begin{aligned} \leqslant & 0.03 \\
\leqslant & 0.015 \\
& 0-06\end{aligned}$ & $\begin{array}{l}0.06 \\
0.06 \\
0.12\end{array}$ & $\begin{array}{l}\leqslant 0.03-0.25 \\
\leqslant 0.015-0.25 \\
\leqslant 0.03-0.5\end{array}$ & $\begin{array}{l}100 \\
100 \\
100\end{array}$ \\
\hline $\begin{array}{l}\text { Klebsiella } \\
\quad \text { pneumoniae (363) }\end{array}$ & $\begin{array}{l}\text { OPC-17116 } \\
\text { ciprofloxacin } \\
\text { temafloxacin }\end{array}$ & 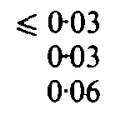 & $\begin{array}{l}0.12 \\
0.25 \\
0.25\end{array}$ & $\begin{array}{l}\leqslant 0.03->4 \\
\leqslant 0.015->2 \\
\leqslant 0.03->4\end{array}$ & $\begin{array}{l}99 \\
97 \\
98\end{array}$ \\
\hline $\begin{array}{l}\text { Morganella } \\
\quad \text { morganni (72) }\end{array}$ & $\begin{array}{l}\text { OPC- } 17116 \\
\text { ciprofloxacin } \\
\text { temafloxacin }\end{array}$ & $\begin{array}{c}0.12 \\
\leqslant 0.015 \\
0.25\end{array}$ & $\begin{array}{l}0.25 \\
0.12 \\
1\end{array}$ & $\begin{array}{l}\leqslant 0.03->4 \\
\leqslant 0.015->2 \\
\leqslant 0.03->4\end{array}$ & $\begin{array}{l}94 \\
94 \\
93\end{array}$ \\
\hline $\begin{array}{l}\text { Proteus mirabilis } \\
\quad \text { (184) }\end{array}$ & $\begin{array}{l}\text { OPC-17116 } \\
\text { ciprofloxacin } \\
\text { temafloxacin }\end{array}$ & $\begin{array}{l}0.25 \\
0.03 \\
0.25\end{array}$ & $\begin{array}{l}0.5 \\
0.06 \\
0.5\end{array}$ & $\begin{array}{l}\leqslant 0.03->4 \\
\leqslant 0.015->2 \\
\leqslant 0.03->4\end{array}$ & $\begin{array}{l}97 \\
97 \\
97\end{array}$ \\
\hline $\begin{array}{l}\text { Proteus vulgaris } \\
\text { (22) }\end{array}$ & $\begin{array}{l}\text { OPC- } 17116 \\
\text { ciprofloxacin } \\
\text { temafloxacin }\end{array}$ & $\begin{array}{l}0.06 \\
\leqslant 0.015 \\
0.25\end{array}$ & $\begin{array}{l}0.5 \\
0.06 \\
1\end{array}$ & $\begin{array}{l}\leqslant 0.03->4 \\
\leqslant 0.015->2 \\
\leqslant 0.03->4\end{array}$ & $\begin{array}{l}95 \\
95 \\
95\end{array}$ \\
\hline P. rettgeri $(17)$ & $\begin{array}{l}\text { OPC-17116 } \\
\text { ciprofloxacin } \\
\text { temafloxacin }\end{array}$ & $\begin{array}{l}4 \\
2 \\
2\end{array}$ & $\begin{array}{l}>4 \\
>2 \\
>4\end{array}$ & $\begin{array}{l}\leqslant 0-03->4 \\
\leqslant 0-015->2 \\
\leqslant 0-03->4\end{array}$ & $\begin{array}{l}47 \\
47 \\
53\end{array}$ \\
\hline
\end{tabular}


Table I.-continued

\begin{tabular}{|c|c|c|c|c|c|}
\hline $\begin{array}{l}\text { Organism } \\
\text { (no. of isolates) }\end{array}$ & $\begin{array}{c}\text { Antimicrobial } \\
\text { agent }\end{array}$ & $\mathrm{MIC}_{50}$ & $\mathrm{MIC}_{90}$ & Range & $\begin{array}{c}\text { Susceptibility } \\
(\%)\end{array}$ \\
\hline P. stuartii (13) & $\begin{array}{l}\text { OPC-17116 } \\
\text { ciprofloxacin } \\
\text { temafloxacin }\end{array}$ & $\begin{array}{l}2 \\
0-5 \\
2\end{array}$ & $\begin{array}{l}>4 \\
>2 \\
>4\end{array}$ & $\begin{aligned} \leqslant & 0.03->4 \\
\leqslant & 0 \cdot 015->2 \\
& 0 \cdot 12->4\end{aligned}$ & $\begin{array}{l}62 \\
54 \\
54\end{array}$ \\
\hline $\begin{array}{l}\text { Salmonella spp. } \\
(35)\end{array}$ & $\begin{array}{l}\text { OPC-17116 } \\
\text { ciprofloxacin } \\
\text { temafloxacin }\end{array}$ & $\begin{array}{c}\leqslant 0.03 \\
\leqslant 0.015 \\
0.12\end{array}$ & $\begin{array}{l}0-06 \\
0.03 \\
0.12\end{array}$ & $\begin{array}{l}\leqslant 0.03-0.12 \\
\leqslant 0.015-0.06 \\
\leqslant 0.03-0.25\end{array}$ & $\begin{array}{l}100 \\
100 \\
100\end{array}$ \\
\hline $\begin{array}{l}\text { Serratia liquefaciens } \\
\quad(25)\end{array}$ & $\begin{array}{l}\text { OPC-17116 } \\
\text { ciprofloxacin } \\
\text { temafloxacin }\end{array}$ & $\begin{array}{l}0.12 \\
0.12 \\
0.25\end{array}$ & $\begin{array}{r}4 \\
>2 \\
>4\end{array}$ & $\begin{aligned} \leqslant & 0.03->4 \\
\leqslant & 0.015->2 \\
& 0.06->4\end{aligned}$ & $\begin{array}{l}80 \\
72 \\
68\end{array}$ \\
\hline S. marcescens (75) & $\begin{array}{l}\text { OPC-17116 } \\
\text { ciprofloxacin } \\
\text { temafloxacin }\end{array}$ & $\begin{array}{l}0-25 \\
0-12 \\
1\end{array}$ & $\begin{array}{l}>4 \\
>2 \\
>4\end{array}$ & $\begin{aligned} \leqslant & 0.03->4 \\
\leqslant & 0-015->2 \\
& 0-12->4\end{aligned}$ & $\begin{array}{l}80 \\
79 \\
68\end{array}$ \\
\hline Serratia spp. (32) & $\begin{array}{l}\text { OPC- } 17116 \\
\text { ciprofloxacin } \\
\text { temafloxacin }\end{array}$ & $\begin{array}{l}0.25 \\
0.12 \\
0.5\end{array}$ & $\begin{array}{l}>4 \\
>2 \\
>4\end{array}$ & $\begin{array}{c}\leqslant 0.03->4 \\
\leqslant 0.015->2 \\
0.06->4\end{array}$ & $\begin{array}{l}73 \\
67 \\
64\end{array}$ \\
\hline $\begin{array}{l}\text { Other Entero- } \\
\text { bacteriaceae' (33) }\end{array}$ & $\begin{array}{l}\text { OPC- } 17116 \\
\text { ciprofloxacin } \\
\text { temafloxacin }\end{array}$ & $\begin{array}{l}\leqslant 0.03 \\
\leqslant 0-015 \\
0.06\end{array}$ & $\begin{array}{l}0.12 \\
0.12 \\
0.5\end{array}$ & $\begin{array}{l}\leqslant 0.03-0.25 \\
\leqslant 0.015-1 \\
\leqslant 0.03-1\end{array}$ & $\begin{array}{l}100 \\
100 \\
100\end{array}$ \\
\hline $\begin{array}{l}\text { Aeromonas } \\
\quad \text { hydrophila (11) }\end{array}$ & $\begin{array}{l}\text { OPC-17116 } \\
\text { ciprofloxacin } \\
\text { temafloxacin }\end{array}$ & $\begin{array}{c}0.06 \\
\leqslant 0.015 \\
0.06\end{array}$ & $\begin{array}{l}0-5 \\
0.5 \\
1\end{array}$ & $\begin{array}{l}\leqslant 0.03-2 \\
\leqslant 0.015-1 \\
\leqslant 0.03-2\end{array}$ & $\begin{array}{l}100 \\
100 \\
100\end{array}$ \\
\hline A. baumannii (38) & $\begin{array}{l}\text { OPC-17116 } \\
\text { ciprofloxacin } \\
\text { temafloxacin }\end{array}$ & $\leqslant \begin{array}{l}0.03 \\
0-2 \\
0-06\end{array}$ & $\begin{array}{l}>4 \\
>2 \\
>4\end{array}$ & $\begin{array}{l}\leqslant 0.03->4 \\
\leqslant 0.015->2 \\
\leqslant 0.03->4\end{array}$ & $\begin{array}{l}84 \\
74 \\
82\end{array}$ \\
\hline $\begin{array}{l}\text { Acinetobacter lwoffi } \\
\text { (11) }\end{array}$ & $\begin{array}{l}\text { OPC-17116 } \\
\text { ciprofloxacin } \\
\text { temafloxacin }\end{array}$ & $\leqslant \begin{array}{r}0.03 \\
0-06 \\
0-06\end{array}$ & $\begin{array}{l}0.06 \\
0.12 \\
0.12\end{array}$ & $\begin{array}{l}\leqslant 0.03-0-06 \\
\leqslant 0-015-0.12 \\
\leqslant 0.03-0.25\end{array}$ & $\begin{array}{l}100 \\
100 \\
100\end{array}$ \\
\hline $\begin{array}{l}\text { Acinetobacter spp. } \\
\text { (12) }\end{array}$ & $\begin{array}{l}\text { OPC- } 17116 \\
\text { ciprofloxacin } \\
\text { temafloxacin }\end{array}$ & $\begin{array}{l}\leqslant 0.03 \\
0.06 \\
\leqslant 0.03\end{array}$ & $\begin{array}{l}>4 \\
>2 \\
>4\end{array}$ & $\begin{array}{l}\leqslant 0-03->4 \\
\leqslant 0-015->2 \\
\leqslant 0-03->4\end{array}$ & $\begin{array}{l}83 \\
75 \\
83\end{array}$ \\
\hline P. aeruginosa $(70)$ & $\begin{array}{l}\text { OPC-17116 } \\
\text { ciprofloxacin } \\
\text { temafloxacin }\end{array}$ & $\begin{array}{l}0-5 \\
0-25 \\
1\end{array}$ & $\begin{array}{l}>4 \\
>2 \\
>4\end{array}$ & $\begin{array}{l}\leqslant 0-03->4 \\
\leqslant 0-015->2 \\
\leqslant 0-03->4\end{array}$ & $\begin{array}{l}74 \\
79 \\
69\end{array}$ \\
\hline Pseudomonas spp. & $\begin{array}{l}\text { OPC-17116 } \\
\text { ciprofloxacin } \\
\text { temafloxacin }\end{array}$ & $\begin{array}{l}0.06 \\
0.25 \\
0.25\end{array}$ & $\begin{array}{l}1 \\
1 \\
2\end{array}$ & $\begin{array}{l}\leqslant 0-03->4 \\
\leqslant 0-015->2 \\
\leqslant 0.03->4\end{array}$ & $\begin{array}{l}95 \\
90 \\
90\end{array}$ \\
\hline X. maltophilia (61) & $\begin{array}{l}\text { OPC-17116 } \\
\text { ciprofloxacin } \\
\text { temafloxacin }\end{array}$ & $\begin{array}{l}0.25 \\
1 \\
0.5\end{array}$ & $\begin{array}{r}2 \\
>2 \\
4\end{array}$ & $\begin{array}{l}\leqslant 0.03->4 \\
\leqslant 0.015->2 \\
\leqslant 0.03->4\end{array}$ & $\begin{array}{l}90 \\
51 \\
85\end{array}$ \\
\hline
\end{tabular}


Table I.-continued

\begin{tabular}{|c|c|c|c|c|c|}
\hline $\begin{array}{l}\text { Organism } \\
\text { (no. of isolates) }\end{array}$ & $\begin{array}{l}\text { Antimicrobial } \\
\text { agent }\end{array}$ & $\mathrm{MIC}_{50}$ & $\mathrm{MIC}_{\mathbf{s}_{0}}$ & Range & $\begin{array}{c}\text { Susceptibility } \\
(\%)\end{array}$ \\
\hline $\begin{array}{l}\text { Flavobacterium spp. } \\
\quad(10)\end{array}$ & $\begin{array}{l}\text { OPC-17116 } \\
\text { ciprofloxacin } \\
\text { temafloxacin }\end{array}$ & $\begin{array}{l}0.5 \\
2 \\
1\end{array}$ & $\begin{array}{l}>4 \\
>2 \\
>4\end{array}$ & $\begin{array}{c}\leqslant 0.03->4 \\
0.5->2 \\
0.25->4\end{array}$ & $\begin{array}{l}70 \\
40 \\
50\end{array}$ \\
\hline $\begin{array}{l}\text { Other non-enteric } \\
\text { species }{ }^{\circ}(18)\end{array}$ & $\begin{array}{l}\text { OPC-17116 } \\
\text { ciprofloxacin } \\
\text { temafloxacin }\end{array}$ & $\begin{array}{l}0.5 \\
0.5 \\
0.25\end{array}$ & $\begin{array}{l}>4 \\
>2 \\
>4\end{array}$ & $\begin{array}{l}\leqslant 0.03->4 \\
\leqslant 0.015->2 \\
\leqslant 0.03->4\end{array}$ & $\begin{array}{l}78 \\
72 \\
67\end{array}$ \\
\hline
\end{tabular}

${ }^{-S}$ See text.

Table II. MICs (mg/L) of OPC-17116, ciprofloxacin and temafloxacin against Gram-positive clinical isolates

\begin{tabular}{|c|c|c|c|c|c|}
\hline $\begin{array}{l}\text { Organism } \\
\text { (no. of isolates) }\end{array}$ & $\begin{array}{c}\text { Antimicrobial } \\
\text { agent }\end{array}$ & $\mathrm{MIC}_{50}$ & $\mathrm{MIC}_{90}$ & Range & $\begin{array}{c}\text { Susceptibility } \\
(\%)\end{array}$ \\
\hline $\begin{array}{l}\text { Enterococcus avium } \\
\quad(18)\end{array}$ & $\begin{array}{l}\text { OPC-17116 } \\
\text { ciprofloxacin } \\
\text { temafloxacin }\end{array}$ & $\begin{array}{l}0-5 \\
1 \\
1\end{array}$ & $\begin{array}{r}4 \\
>2 \\
>\end{array}$ & $\begin{array}{l}0.12->4 \\
0.25->2 \\
0.5->4\end{array}$ & $\begin{array}{l}83 \\
67 \\
78\end{array}$ \\
\hline E. faecalis (422) & $\begin{array}{l}\text { OPC-17116 } \\
\text { ciprofloxacin } \\
\text { temafloxacin }\end{array}$ & $\begin{array}{l}0 \cdot 5 \\
1 \\
1\end{array}$ & $\begin{array}{l}>4 \\
>2 \\
>4\end{array}$ & $\begin{array}{r}0.06->4 \\
0.03->2 \\
\leqslant 0-03->4\end{array}$ & $\begin{array}{l}70 \\
62 \\
69\end{array}$ \\
\hline E. faecium (56) & $\begin{array}{l}\text { OPC-17116 } \\
\text { ciprofloxacin } \\
\text { temafloxacin }\end{array}$ & $\begin{array}{l}2 \\
1 \\
2\end{array}$ & $\begin{array}{l}>4 \\
>2 \\
>4\end{array}$ & $\begin{array}{l}0-06->4 \\
0 \cdot 12->2 \\
0-06->4\end{array}$ & $\begin{array}{l}61 \\
50 \\
61\end{array}$ \\
\hline $\begin{array}{l}\text { Enterococcus spp. } \\
\quad(176)\end{array}$ & $\begin{array}{l}\text { OPC-17116 } \\
\text { ciprofloxacin } \\
\text { temafloxacin }\end{array}$ & $\begin{array}{l}0 \cdot 12 \\
1 \\
1\end{array}$ & $\begin{array}{r}2 \\
>2 \\
4\end{array}$ & $\begin{array}{l}\leqslant 0.03->4 \\
\leqslant 0.015->2 \\
\leqslant 0.03->4\end{array}$ & $\begin{array}{l}90 \\
81 \\
86\end{array}$ \\
\hline $\begin{array}{l}\text { Streptococcus spp. } \\
\quad(66)\end{array}$ & $\begin{array}{l}\text { OPC-17116 } \\
\text { ciprofloxacin } \\
\text { temafloxacin }\end{array}$ & $\begin{array}{l}0 \cdot 12 \\
1 \\
0 \cdot 5\end{array}$ & $\begin{array}{l}0-5 \\
2 \\
1\end{array}$ & $\begin{array}{l}\leqslant 0-03-4 \\
\leqslant 0-015-2 \\
\leqslant 0-03-2\end{array}$ & $\begin{array}{r}98 \\
94 \\
100\end{array}$ \\
\hline $\begin{array}{l}\beta \text {-Haemolytic } \\
\text { streptococci (14) }\end{array}$ & $\begin{array}{l}\text { OPC-17116 } \\
\text { ciprofloxacin } \\
\text { temafloxacin }\end{array}$ & $\begin{array}{l}0.25 \\
0.5 \\
0.5\end{array}$ & $\begin{array}{l}0-25 \\
1 \\
1\end{array}$ & $\begin{array}{l}0.12-0.5 \\
0.5-1 \\
0.12-1\end{array}$ & $\begin{array}{l}100 \\
100 \\
100\end{array}$ \\
\hline $\begin{array}{l}\text { Oxacillin- } \\
\text { susceptible } \\
\text { S. aureus }(768)\end{array}$ & $\begin{array}{l}\text { OPC-17116 } \\
\text { ciprofloxacin } \\
\text { temafloxacin }\end{array}$ & 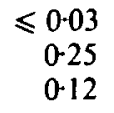 & $\begin{array}{l}0.12 \\
1 \\
0.25\end{array}$ & $\begin{array}{l}\leqslant 0.03->4 \\
\leqslant 0.015->2 \\
\leqslant 0.03->4\end{array}$ & $\begin{array}{l}98 \\
94 \\
97\end{array}$ \\
\hline $\begin{array}{l}\text { Oxacillin-resistant } \\
\text { S. aureus (297) }\end{array}$ & $\begin{array}{l}\text { OPC-17116 } \\
\text { ciprofloxacin } \\
\text { temafloxacin }\end{array}$ & $\begin{array}{l}>4 \\
>2 \\
>4\end{array}$ & $\begin{array}{l}>4 \\
>2 \\
>4\end{array}$ & $\begin{array}{l}\leqslant 0.03->4 \\
\leqslant 0.015->2 \\
\leqslant 0.03->4\end{array}$ & $\begin{array}{l}38 \\
35 \\
37\end{array}$ \\
\hline $\begin{array}{l}\text { Oxacillin-susceptible } \\
\text { S. epidermidis } \\
\text { (164) }\end{array}$ & $\begin{array}{l}\text { OPC-17116 } \\
\text { ciprofloxacin } \\
\text { temafloxacin }\end{array}$ & $\begin{array}{l}0.06 \\
0.25 \\
0.25\end{array}$ & $\begin{array}{l}>4 \\
>2 \\
>4\end{array}$ & $\begin{aligned} \leqslant 0.03->4 \\
0.03->2 \\
\leqslant 0.03->4\end{aligned}$ & $\begin{array}{l}88 \\
85 \\
87\end{array}$ \\
\hline
\end{tabular}


Table II.-continued

\begin{tabular}{|c|c|c|c|c|c|}
\hline $\begin{array}{l}\text { Organism } \\
\text { (no. of isolates) }\end{array}$ & $\begin{array}{l}\text { Antimicrobial } \\
\text { agent }\end{array}$ & $\mathrm{MIC}_{50}$ & $\mathrm{MIC}_{90}$ & Range & $\begin{array}{c}\text { Susceptibility } \\
(\%)\end{array}$ \\
\hline $\begin{array}{l}\text { Oxacillin-resistant } \\
S . \text { epidermidis } \\
\text { (139) }\end{array}$ & $\begin{array}{l}\text { OPC-17116 } \\
\text { ciprofloxacin } \\
\text { temafloxacin }\end{array}$ & $\begin{array}{l}0.12 \\
0.5 \\
0.25\end{array}$ & $\begin{array}{l}>4 \\
>2 \\
>4\end{array}$ & $\begin{array}{r}\leqslant 0.03->4 \\
0.12->2 \\
\leqslant 0-03->4\end{array}$ & $\begin{array}{l}74 \\
64 \\
67\end{array}$ \\
\hline $\begin{array}{c}\text { Staphylococcus } \\
\text { hominis (11) }\end{array}$ & $\begin{array}{l}\text { OPC-17116 } \\
\text { ciprofloxacin } \\
\text { temafloxacin }\end{array}$ & 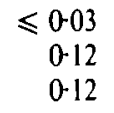 & $\begin{array}{l}0.06 \\
0 \cdot 12 \\
0 \cdot 12\end{array}$ & $\begin{array}{l}\leqslant 0-03->4 \\
0.06->2 \\
\leqslant 0.03-2\end{array}$ & $\begin{array}{r}91 \\
73 \\
100\end{array}$ \\
\hline $\begin{array}{l}\text { Oxacillin- } \\
\text { susceptible } S \text {. } \\
\text { haemolyticus (13) }\end{array}$ & $\begin{array}{l}\text { OPC-17116 } \\
\text { ciprofloxacin } \\
\text { temafloxacin }\end{array}$ & $\begin{array}{l}0 \cdot 06 \\
0 \cdot 12 \\
0-06\end{array}$ & $\begin{array}{l}>4 \\
>2 \\
>4\end{array}$ & $\begin{array}{r}\leqslant 0-03->4 \\
0-06->2 \\
\leqslant 0-03->4\end{array}$ & $\begin{array}{l}85 \\
85 \\
85\end{array}$ \\
\hline $\begin{array}{l}\text { Oxacillin-resistant } \\
\text { S. haemolyticus }\end{array}$ & $\begin{array}{l}\text { OPC-17116 } \\
\text { ciprofloxacin } \\
\text { temafloxacin }\end{array}$ & $\begin{array}{l}>4 \\
>2 \\
>4\end{array}$ & $\begin{array}{l}>4 \\
>2 \\
>4\end{array}$ & $\begin{array}{l}0 \cdot 06->4 \\
0 \cdot 5->2 \\
0 \cdot 12->4\end{array}$ & $\begin{array}{l}21 \\
14 \\
14\end{array}$ \\
\hline $\begin{array}{l}\text { Staphylococcus } \\
\text { saprophyticus } \\
\text { (15) }\end{array}$ & $\begin{array}{l}\text { OPC-17116 } \\
\text { ciprofloxacin } \\
\text { temafloxacin }\end{array}$ & $\begin{array}{l}0.12 \\
0.5 \\
0-5\end{array}$ & $\begin{array}{l}0.12 \\
0.5 \\
0.5\end{array}$ & $\begin{aligned} \leqslant & 0.03-0.25 \\
& 0.12-1 \\
& 0.06-0.05\end{aligned}$ & $\begin{array}{l}100 \\
100 \\
100\end{array}$ \\
\hline $\begin{array}{l}\text { Oxacillin- } \\
\text { susceptible } \\
\text { coagulase- } \\
\text { negative } \\
\text { staphylococci } \\
\text { (133) }\end{array}$ & $\begin{array}{l}\text { OPC-17116 } \\
\text { ciprofloxacin } \\
\text { temafloxacin }\end{array}$ & 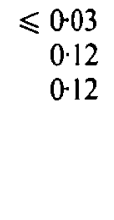 & $\begin{array}{l}0.12 \\
0.5 \\
0.5\end{array}$ & $\begin{array}{l}\leqslant 0-03->4 \\
\leqslant 0-015->2 \\
\leqslant 0-03->4\end{array}$ & $\begin{array}{l}95 \\
93 \\
95\end{array}$ \\
\hline $\begin{array}{l}\text { Oxacillin-resistant } \\
\text { coagulase- } \\
\text { negative } \\
\text { staphylococci } \\
\text { (47) }\end{array}$ & $\begin{array}{l}\text { OPC-17116 } \\
\text { ciprofloxacin } \\
\text { temafloxacin }\end{array}$ & $\begin{array}{l}>4 \\
>2 \\
>4\end{array}$ & $\begin{array}{l}>4 \\
>2 \\
>4\end{array}$ & $\begin{array}{l}\leqslant 0-03->4 \\
\leqslant 0015->2 \\
\leqslant 0.03->4\end{array}$ & $\begin{array}{l}46 \\
42 \\
45\end{array}$ \\
\hline $\begin{array}{l}\text { Staphylococcus } \\
\text { spp." (16) }\end{array}$ & $\begin{array}{l}\text { OPC-17116 } \\
\text { ciprofloxacin } \\
\text { temafloxacin }\end{array}$ & $\begin{array}{l}0.06 \\
0.12 \\
0.12\end{array}$ & $\begin{array}{l}0.25 \\
0.25 \\
0.5\end{array}$ & $\begin{array}{l}\leqslant 0-03->4 \\
0.03-1 \\
\leqslant 0.03->4\end{array}$ & $\begin{array}{r}94 \\
100 \\
94\end{array}$ \\
\hline $\begin{array}{l}\text { Other } \\
\text { Gram-positive } \\
\text { species (18) }\end{array}$ & $\begin{array}{l}\text { OPC-17116 } \\
\text { ciprofloxacin } \\
\text { temafloxacin }\end{array}$ & $\begin{array}{l}0-06 \\
0-12 \\
0-5\end{array}$ & $\begin{array}{l}0.25 \\
1 \\
1\end{array}$ & $\begin{array}{c}\leqslant 0.03-1 \\
0.5-1 \\
0.12-1\end{array}$ & $\begin{array}{l}100 \\
100 \\
100\end{array}$ \\
\hline
\end{tabular}

${ }^{a}$ See text.

Spanish and Japanese participating centres detected more resistant strains than the other countries. Results from Spain are in accordance with a previous communication from this country noting an increase of ciprofloxacin resistance among $E$. coli isolates (Rodriguez-Creixems et al., 1991) and it has been suggested that this could be due to the widespread use of quinolone agents.

Against Acinetobacter baumannii, Xantomonas maltophilia and Flavobaterium spp. OPC-17116 was the most active fluoroquinolone, but only slightly better to temafloxacin. OPC-17116 was, however, less active than ciprofloxacin against $P$. aeruginosa 
isolates. We also tested OPC-17116 against other species of Pseudomonas spp. (21 strains) and 18 strains of other non-enteric species (Table I), including Alcaligenes xylosoxidans subsp xylosoxidans (eight strains), Alcaligenes spp. (five strains), Aeromonas spp. (three strains), Moraxella spp. (one strain) and Pasteurella multocida (one strain). Our data show that OPC-17116 has a greater spectrum of activity against the non-enteric Gram-negative bacilli as a group. Also, it had a greater activity against all species except $P$. aeruginosa that seems to be slightly more susceptibile to ciprofloxacin.

OPC-17116 showed greater antibacterial activity against Gram-positive organisms than ciprofloxacin and temafloxacin. It had a wider spectrum than ciprofloxacin against 14 of 19 Gram-positive organisms and the same against four organisms. The average improved spectrum was $6.4 \%$ (range $2-18 \%$ ). When equally potent as ciprofloxacin, both drugs were usually $100 \%$ effective. OPC- 17116 inhibited $50 \%$ of Enterococcus spp. isolates at MICs of $\leqslant 0.5 \mathrm{mg} / \mathrm{L}$, except Enterococcus faecium isolates that were more resistant to all of the fluoroquinolones tested. The $\mathrm{MIC}_{90}$ for Streptococcus spp., mainly viridans streptococci, was $0.5 \mathrm{mg} / \mathrm{L}$ and all $\beta$-haemolytic streptococci tested were susceptible. OPC-17116 was two- to three-fold more active than ciprofloxacin against oxacillin-susceptible isolates of $S$. aureus, Staphylococcus epidermidis and other coagulase-negative staphylococci. However, when these species were oxacillin-resistant, the activity of OPC-17116 was only slightly better than ciprofloxacin. Oxacillin-resistant $S$. aureus isolates are a serious problem in many hospitals around the world. Rapid development of resistance to fluoroquinolones in oxacillin-susceptible and -resistant $S$. aureus has been reported previously (Blumberg $e t$ al., 1991). Against these isolates, Wakeba \& Mitsuhashi (1992) found different percentages of resistance to quinolones suggesting that OPC-17116 showed incomplete crossresistance compared with ciprofloxacin. However, in our study, the activity of OPC-17116 against oxacillin-resistant $S$. aureus was comparable to or only slightly better than those of the other quinolones tested.

Oxacillin-resistant Staphylococcus hemolyticus was the most resistant species to all fluoroquinolones studied. OPC-17116 inhibited $90 \%$ of other Staphylococcus spp. tested at $\leqslant 0.25 \mathrm{mg} / \mathrm{L}$ including Staphylococcus warneri (five strains), Staphyloccoccus simulans (three strains), Staphylococcus auricularis (three strains), Staphylococcus intermedius (two strains), and one strain each of Staphylococcus capitis, Staphylococcus sciuri and Staphylococcus cohnii. All strains of other Gram-positive species tested such as Bacillus spp. (11 strains) and Micrococcus spp. (seven strains) were susceptible to the three quinolones studied.

Finally, OPC-17116 clearly exhibited a wider spectrum against comtemporary pathogens than ciprofloxacin in the strains from the six countries. In view of the in-vitro activity demonstrated in this study, together with results reported elsewhere against potential respiratory pathogens (Wakebe \& Mitsuhashi, 1992) and its pharmacokinetic properties (Akiyama et al., 1991), OPC-17116 may be an alternative antimicrobial agent for some serious infections. Accordingly, comparative clinical trials as well as studies of toxicity and long-term side effects are warranted.

\section{Acknowledgements}

We wish to thank the technical support of $\mathrm{M}$. Parera (Microbiology Laboratory, Hospital Clinic, Barcelona) and $\mathrm{S}$. Anderson for data processing (Iowa ARC Laboratory). 


\section{References}

Akiyama, H., Koike, M., Nii, S., Ohguro, K. \& Odomi, M. (1991). OPC-17116, an excellently tissue-penetrative new quinolone: pharmacokinetic profiles in animals and antibacterial activities of metabolites. In Program and Abstracts of the Thirty-First Interscience Conference on Antimicrobial Agents and Chemotherapy, Chicago, 1991. Abstract 1477, p. 345. American Society for Mircobiology, Washington, DC.

Blumberg, H. M., Rimland, D., Carroll, D. J., Terry, P. \& Wachsmuth, I. K. (1991). Rapid development of ciprofloxacin resistance in methicillin-susceptible and -resistant Staphylococcus aureus. Journal of Infectious Diseases 163, 1279-85.

Imada, T., Miyazaki, S., Nishida, M., Yamaguchi, K. \& Goto, S. (1992). In vitro and in vivo antibacterial activities of a new quinolone, OPC-17116. Antimicrobial Agents and Chemotherapy 36, 573-9.

Jones, R. N., Reller, L. B., Rosati, L. A., Erwin, M. E., Sanchez, M. L. \& the Ofloxacin Surveillance Group. (1992). Ofloxacin, a new broad-spectrum fluoroquinolone. Results from a multicenter, national comparative activity surveillance study. Diagnostic Microbiology and Infectious Diseases 15, 425-34.

National Committee for Clinical Laboratory Standards. (1990). Methods for Dilution Antimicrobial Susceptibility Test for Bacteria That Grow Aerobically, Second Edition; Approved Standard M7-A2. NCCLS, Villanova, PA.

Richard, P. \& Gutmann, L. (1992). Sparfloxacin and other new fluoroquinolones. Journal of Antimicrobial Chemotherapy 30, 739-44.

Rodriguez-Creixems, M., Diaz, M. D., Muñoz, P., Baraia, J., Cercenado, E. \& Bouza, E. (1991). Emergence of clinical isolates of Escherichia coli resistant to ciprofloxacin. In Program and Abstracts of the Thirty-First Interscience Conference on Antimicrobial Agents and Chemotherapy, Chicago, 1991. Abstract 138, p. 121. American Society for Microbiology, Washington, DC.

Sader, H. S., Erwin, M. E. \& Jones, R. N. (1992). In-vitro activity of OPC-17116 compared to other broad-spectrum fluoroquinolones. European Journal of Clinical Microbiology and Infectious Diseases 11, 372-81.

Wakebe, H. \& Mitsuhashi, S. (1992). Comparative in-vitro activities of a new quinolone, OPC-17116, possessing potent activity against Gram-positive bacteria. Antimicrobial Agents and Chemotherapy 36, 2185-91.

Yokota, T., Arai, K. \& Kanda, K. (1991). In vitro activity of OPC-17116, a 4-quinolone newly synthesized and possessing a methyl moiety at the 5 position. In Program and Abstracts of the Thirty-First Interscience Conference on Antimicrobial Agents and Chemotherapy, Chicago, 1991. Abstract 1462, p. 342. American Society for Microbiology, Washington, DC.

(Received 22 March 1993; revised version accepted I November 1993) 